\title{
MAPCUMBA: A fast iterative multi-grid map-making algorithm for CMB experiments
}

\author{
O. Doré ${ }^{1}$, R. Teyssier ${ }^{1,2}$, F. R. Bouchet ${ }^{1}$, D. Vibert ${ }^{1}$, and S. Prunet ${ }^{3}$ \\ 1 Institut d'Astrophysique de Paris, 98bis, Boulevard Arago, 75014 Paris, France \\ 2 Service d'Astrophysique, DAPNIA, Centre d'Études de Saclay, 91191 Gif-sur-Yvette, France \\ 3 CITA, McLennan Labs, 60 St George Street, Toronto, ON M5S 3H8, Canada
}

Received 21 December 2000 / Accepted 2 May 2001

\begin{abstract}
The data analysis of current Cosmic Microwave Background (CMB) experiments like BOOMERanG or MAXIMA poses severe challenges which already stretch the limits of current (super-) computer capabilities, if brute force methods are used. In this paper we present a practical solution for the optimal map making problem which can be used directly for next generation CMB experiments like ARCHEOPS and TopHat, and can probably be extended relatively easily to the full PLANCK case. This solution is based on an iterative multi-grid Jacobi algorithm which is both fast and memory sparing. Indeed, if there are $\mathcal{N}_{\text {tod }}$ data points along the one dimensional timeline to analyse, the number of operations is of $\mathcal{O}\left(\mathcal{N}_{\text {tod }} \ln \mathcal{N}_{\text {tod }}\right)$ and the memory requirement is $\mathcal{O}\left(\mathcal{N}_{\text {tod }}\right)$. Timing and accuracy issues have been analysed on simulated ARCHEOPS and TopHat data, and we discuss as well the issue of the joint evaluation of the signal and noise statistical properties.
\end{abstract}

Key words. methods: data analysis - cosmic microwave background

\section{Introduction}

As cosmology enters the era of "precision", it enters simultaneously the era of massive data sets. This has in turn showed the need for new data processing algorithms. Present and future CMB experiments in particular face some new and interesting challenges (Bond et al. 1999). If we accept the now classical point of view of a four steps data analysis pipeline: i) from time-ordered data (TOD) to maps of the sky at a given frequency, ii) from frequency maps to (among others) a temperature map, iii) from a temperature map to its power spectrum $C_{\ell}$, iv) from the power spectrum to cosmological parameters and characteristics of the primordial spectrum of fluctuation, the ultimate quantities to be measured in a given model. The work we are presenting focuses on the first of these issues, namely the map-making step.

Up to the most recent experiments, maps could be made by a brute force approach that aims to solve directly a large linear problem by direct matrix inversion. Nevertheless the size of the problem, and the required computing power, grows as $\mathcal{O}\left(N_{\text {pix }}^{3}\right)$, and the limits of this kind of method have now been reached (Borrill 2000) (even if the $\mathcal{O}\left(N_{\text {pix }}^{2}\right)$ scaling of a preconditioned conjuguate gradient is actually the appropirate scaling for mapmaking only). Whereas the most efficient development

Send offprint requests to: O. Doré, e-mail: dore@iap.fr in this massive computing approach, i.e. the MADCAP package (Borrill 1999) has been applied to the recent BOOMERanG and MAXIMA experiments (de Bernardis et al. 2000; Hanany et al. 2000) some faster and less consuming solutions based on iterative inversion algorithms have now been developed and applied too (Prunet et al. 2000). This is in the same spirit as (Wright et al. 1996), and we definitely follow this latter approach.

Design goals are to use memory sparingly by handling only columns or rows instead of full matrixes and to increase speed by minimising the number of iterations required to reach the sought convergence of the iterative scheme. We fulfill these goals by an iterative multi-grid Jacobi algorithm. As recalled below, an optimal method involves using the noise power spectrum. We have thus investigated the possibility of a joint noise (and signal) evaluation using this algorithm.

Section 2 presents in detail the method and its implementation, while Sect. 3 demonstrates its capabilities by using simulations of two on-going balloon CMB experiments, ARCHEOPS ${ }^{1}$ (Benoit et al. 2000) and TopHat ${ }^{2}$. The results are discussed in Sect. 4, together with the problem of the evaluation of the noise properties, as well as possible extensions.

\footnotetext{
1 http://www-crtbt.polycnrs-gre.fr/archeops/

2 http://topweb.gsfc.nasa.gov/
} 


\section{The method}

\subsection{Optimal map making}

The relation between the sky map we seek and the observed data stream may be cast as a linear algebra system (Wright et al. 1996; Tegmark 1997; Bond et al. 1998). Let $t$ and $\mathrm{p}$ indices denote quantities in the temporal and spatial domains, and group as a data vector, $d_{\mathrm{t}}$, and a noise vector the temporal stream of collected data and the detector noise stream, both of dimension $\mathcal{N}_{\text {tod }}$. We then have

$d_{\mathrm{t}}=A_{\mathrm{tp}} x_{\mathrm{p}}+n_{\mathrm{t}}$,

where $A_{\mathrm{tp}} x_{\mathrm{p}}$ is the signal vector given by the observation of the unknown pixelised sky map, $x_{\mathrm{p}}$, which has been arranged as a vector of dimension $\mathcal{N}_{\text {pix }}$. The $\mathcal{N}_{\text {tod }} \times \mathcal{N}_{\text {pix }}$ "observation" matrix $A$ therefore encompasses the scanning strategy and the beam pattern of the detector.

In the following, we restrict the discussion to the case when the beam pattern is symmetrical. We can therefore take $x_{\mathrm{p}}$ to be a map of the sky which has already been convolved with the beam pattern, and $A$ only describes how this sky is being scanned. For the total power measurement (i.e. non-differential measurement) we are interested in here, the observation matrix $A$ then has a single non-zero element per row, which can be set to one if $d$ and $x$ are expressed in the same units. The model of the measurement is then quite transparent: each temporal datum is the sum of the pixel value to which the detector is pointing plus the detector noise.

The map-making step then amounts to best solve for $x$ given $d$ (and some properties of the noise). We shall seek a linear estimator of $x_{\mathrm{p}}$,

$\hat{x}_{\mathrm{p}}=W_{\mathrm{pt}} d_{\mathrm{t}}$.

To motivate a particular choice of the $\mathcal{N}_{\text {pix }} \times \mathcal{N}_{\text {tod }}$ matrix $W$, a Bayesian approach is convenient. Indeed we are seeking the optimal solution to this inversion problem which maximises the probability of a deduced set of theory parameters (here the map $x_{\mathrm{p}}$ ) given our data $\left(d_{\mathrm{t}}\right)$ by maximising $\mathcal{P}(x \mid d)$. Bayes' theorem simply states that

$\mathcal{P}(x \mid d)=\frac{\mathcal{P}(d \mid x) \mathcal{P}(x)}{\mathcal{P}(d)}$.

If we do not assume any theoretical prior, then $x$ follows a uniform distribution as well as $d$. Therefore,

$\mathcal{P}(x \mid d) \propto \mathcal{P}(d \mid x)$.

If we further assume that the noise is Gaussian, we can write

$$
\begin{aligned}
\mathcal{P}(x \mid d) & \propto \exp \left(-n_{\mathrm{t}}^{T} N_{\mathrm{tt}^{\prime}}^{-1} n_{\mathrm{t}^{\prime}} / 2\right) \\
& \propto \exp \left(-(d-A x)_{\mathrm{t}}^{T} N_{\mathrm{tt}^{\prime}}^{-1}(d-A x)_{\mathrm{t}^{\prime}} / 2\right) \\
& \propto \exp \left(-\chi^{2} / 2\right)
\end{aligned}
$$

where $N_{\mathrm{tt}^{\prime}}=\left\langle n n^{T}\right\rangle_{\mathrm{tt}^{\prime}}$ is the noise covariance matrix. In this particular case, maximising $\mathcal{P}(x \mid d)$ amounts to find the least square solution which was used to analyse the COBE data (Jansen \& Gulkis 1992),

$W=\left[A^{T} N^{-1} A\right]^{-1} A^{T} N^{-1}$

In this paper we will actually deal only with this estimator. Nevertheless as a next iteration in the analysis process, we could incorporate various theoretical priors by making $\mathcal{P}(x)$ more explicit. For example, it is often assumed a Gaussian prior for the theory, i.e. $\mathcal{P}(x) \propto$ $\exp \left(-x_{\mathrm{p}}^{T} C_{\mathrm{pp}^{\prime}}^{-1} x_{\mathrm{p}^{\prime}} / 2\right)$ where $C_{\mathrm{pp}^{\prime}}=\left\langle x_{\mathrm{p}} x_{\mathrm{p}^{\prime}}^{T}\right\rangle$ is the signal covariance matrix. In that case the particular solution turns out to be the Wiener filtering solution (Zaroubi et al. 1995; Bouchet \& Gispert 1996; Tegmark \& Efstathiou 1996; Bouchet \& Gispert 1998):

$W=\left[C^{-1}+A^{T} N^{-1} A\right]^{-1} A^{T} N^{-1}$.

However this solution may always be obtained by a further (Wiener) filtering of the priorless maximum likelihood solution, and we do not consider it further.

The prior-less solution demonstrates that as long as the (Gaussian) instrumental noise is not white, a simple averaging (co-addition) of all the data points corresponding to a given sky pixel is not optimal. If the noise exhibits some temporal correlations, as induced for instance by a low-frequency $1 / f$ behavior of the noise spectrum which prevails in most CMB experiments, one has to take into account the full time correlation structure of the noise. Additionally, this expression demonstrates that even if the noise has a simple time structure, the scanning strategy generically induces a non-trivial correlation matrix $\left[A^{T} N^{-1} A\right]^{-1}$ of the noise map.

Even if the problem is well posed formally, a quick look at the orders of magnitude shows that the actual finding of a solution is a non trivial task. Indeed, a brute force method aiming at inverting the full matrix $\left[A^{T} N^{-1} A\right]^{-1}$, an operation scaling as $\mathcal{O}\left(\mathcal{N}_{\text {pix }}^{3}\right)$, is already hardly tractable for present long duration balloon flights as MAXIMA, BOOMERanG, ARCHEOPS or TopHat where $\mathcal{N}_{\text {tod }} \sim 10^{7}$ and $\mathcal{N}_{\text {pix }} \sim 10^{5}$. It appears totally impractical for PLANCK since for a single detector (amid 10s) $\mathcal{N}_{\text {tod }} \sim 10^{9}$ and $\mathcal{N}_{\text {pix }} \sim 10^{7}$ !

One possibility may be to take advantage of specific scanning strategies, and actually solve the inverse of the convolution problem as detailed in Wandelt \& Gorski (2000). This amounts to deducing the map coefficients $a_{\mathrm{lm}}$ in the spherical harmonic basis through a rotation of a Fourier decomposition of the observed data. The map will then be a simple visualisation device, while the $a_{\mathrm{lm}}$ would be used directly for a component separation (as in Bouchet \& Gispert 1996; Tegmark \& Efstathiou 1996; Bouchet \& Gispert 1998) and the CMB power spectrum estimate. While potentially very interesting, this approach will not be generally applicable (at least efficiently), and we now turn to a practical (general) solution of Eq. (2) by iterative means. 


\subsection{Practical implementation for large data sets}

We solve the map-making problem by adapting to our particular case the general "multi-grid method" (Press et al. 1992). Multi-grid methods are commonly used to speed up the convergence of a traditional relaxation method (in our case the Jacobi method, as in Prunet et al. 2000 defined at resolution $\ell_{\max }$ (see below). A set of recursively defined coarser grids $\left(\ell<\ell_{\max }\right)$ are used as temporary computational space, in order to increase the convergence rate of the relaxation process. To be fully profitable, this algorithm implies for each resolution both a rebinning in space (resolution change) and in time (resampling).

In this paper, we use the HEALPix pixelisation of the sphere (Górski et al. 1998). In this scheme, the sphere is covered by 12 basic quadrilaterals, further divided recursively into pixels of equal area. The map resolution is labeled by $N_{\text {side }}$ : the number of pixels along the side of one basic quadrilateral. Hence, $N_{\text {side }}=1$ means that the sphere is covered by 12 large pixels only. The number of pixels is given by $N_{\text {pix }}=12 N_{\text {side }}^{2} \cdot N_{\text {side }}=256$ corresponds to a pixel size of 13.7 arcmin. For practical reasons, we need to define the resolution $k$ of a HEALPix map as

$N_{\text {side }}=2^{k}$.

The "nested" pixel numbering scheme of HEALPix (Górski et al. 1998) allows an easy implementation of the coarsening $(k \rightarrow k-1)$ and refining $(k \rightarrow k+1)$ operators that we use intensively in our multigrid scheme.

We now discuss the details of our implementation and of the exact system we solve, the way we solve it and the actual steps of the multi-grid algorithm.

\subsubsection{Determining the working resolution $k_{\max }$}

The timeline is given by $d=A_{\infty} x_{\infty}+n$ where $x_{\infty}$ is the sky map at "infinite" resolution $(k=+\infty$ in our notations). We aim at solving for the optimal map $\hat{x}_{k}$ at a given finite spatial resolution $k$ using

$A_{k}^{T} N^{-1} A_{k} \hat{x}_{k}=A_{k}^{T} N^{-1} d$,

where $A_{k}$ is the "observation" operator (from spatial to temporal domain) and $A_{k}^{T}$ is the "projection" operator (from temporal to spatial domain). In a noise-free experiment, the optimal map would be straightforwardly given by the co-added map (introducing the "co-addition" operator $P_{k}$ )

$\hat{x}_{k}=P_{k} d \equiv\left(A_{k}^{T} A_{k}\right)^{-1} A_{k}^{T} d$.

In order to check the accuracy of this trivial noise-free map making, it is natural to compute the residual in the time domain with $n=0$

$p_{k}=A_{k} \hat{x}_{k}-d=A_{k} \hat{x}_{k}-A_{\infty} x_{\infty}$

which will be non-zero in practice, as soon as one works with finite spatial resolution. We call this residual the pixelisation noise. Since we assume here that the instrumental beam is symmetric, the sky map is considered as the true sky convolved by, say, a Gaussian beam of angular diameter $\Delta \theta_{\mathrm{B}}$. This introduces a low-pass spatial filter in the problem. In other words, as the resolution increases, the pixelisation noise should decrease towards zero. We evaluate the order of magnitude of the pixelisation noise this way:

$$
\begin{aligned}
\left\|p_{k}\right\| & \simeq\left\|\frac{\Delta x}{\Delta \theta}\right\|_{\infty} \Delta \theta_{k} \\
& \leq \frac{\|x\|_{\infty}}{\Delta \theta_{B}} \Delta \theta_{k}
\end{aligned}
$$

where $\Delta \theta_{k}$ is the typical pixel width at resolution $k$ and $\|x\|_{\infty}$ is the signal norm. This formula is an evaluation of the maximal variation of the signal visible on a beam size. The norms used in the above formula can be either the maximum over the time line (a very strong constraint) or the variance over the time line (a weaker constraint). Since the pixelisation noise is strongly correlated with the sky signal (the more the signal varies, the higher this noise), point sources or Galaxy crossings are potential candidates for large and localised bursts of pixelisation noise. The correct working resolution $k_{\max }$ is set by requiring that the pixelisation noise remains low compared to the actual instrumental noise, or equivalently

$\Delta \theta_{\mathrm{B}} \leq \frac{\Delta \theta_{k_{\max }}}{S / N}$

Note however that the pixelisation noise is strongly non Gaussian (point sources or Galaxy crossings) and can be always considered as a potential source of residual stripes in the final maps.

\subsubsection{The basic relaxation method: An approximate Jacobi solver}

Instead of solving for $\hat{x}$ we perform the change of variable

$\hat{y}=\hat{x}-P d$

and solve instead for $\hat{y}$ which obeys

$P N^{-1} A \hat{y}=P N^{-1}(d-A P d) \quad$ or $\quad M \hat{y}=b$

where we have multiplied each side of Eq. (11) by $\left(A^{T} A\right)^{-1}$, the pixel hit counter. From now on, we also assume that the noise in the timestream is stationary and that its covariance matrix is normalised so that $\operatorname{diag} N_{\mathrm{tt}^{\prime}}^{-1}=I$. The previous change of variable allows us to subtract the sky signal from the data: since we have chosen a resolution high enough to neglect the pixelisation noise, we have indeed $A P d \simeq A_{\infty} x_{\infty}+A P n$ and, consequently, $d-A P d \simeq n-A P n$. The map making consists of two steps: first to compute a simple co-added map from the time line, and second, to solve Eq. (18) for the stripes map $\hat{y}$. The final optimal map is obtained by adding these two maps.

It is worth mentioning that the stripes map is completely independent of the sky signal, as soon as the pixelisation noise can be ignored. It depends only on the scanning strategy through the matrix $A$ and on the noise 
characteristics through the matrix $N$. Even if in principle this change of variable is irrelevant since it does not change the matrix to be inverted, it does so in practice since $d-A P d$ is free from the fast variations of $d$ (up to the pixelisation noise), as e.g. the Galaxy crossings, which are numerically damaging to the quality of the inversion.

To solve for Eq. (18), we follow the ideas of Prunet et al. (2000) and apply an approximate Jacobi relaxation scheme. The Jacobi method consists in the following relaxation step to solve for our problem

$\hat{y}^{n+1}=R \hat{y}^{n}+D^{-1} b \quad$ and $\quad \hat{y}^{0}=0$

where $D$ is the diagonal part of the matrix $M$ and $R$ is the residual matrix $R=I-D^{-1} M$. Computing the diagonal elements of $M=P N^{-1} A$ is rather prohibitive. The idea of Prunet et al. (2000) is to approximate $D \simeq I$ by neglecting the off-diagonal elements of $N^{-1}$. The residual matrix then simplifies greatly and writes

$R=P\left(I-N^{-1}\right) A$.

The approximate Jacobi relaxation step is therefore defined as

$\hat{y}^{n+1}=R \hat{y}^{n}+b \quad$ and $\quad \hat{y}^{0}=0$.

One clearly sees that if this iterative scheme converges, it is towards the full solution of Eq. (18). To perform these successive steps, it is extremely fruitful to remember the assumed stationarity of the noise. Indeed whereas this assumption implies a circulant noise covariance matrix in real space, it translates in Fourier space in the diagonality of the noise covariance matrix. This is naturally another formulation of the convolution theorem, since a stationary matrix acts on a vector as a convolution, and a diagonal matrix acts as a simple vector product, thus a convolution in real space is translated as a product in Fourier space. The point is that the manipulation of the matrix $N^{-1}$ is considerably lighter and will henceforth be performed in Fourier space. Applying the matrix $R$ to a map reduces then to the following operations in order to:

1. "Observe" the previous stripes map $\hat{y}^{n}$;

2. Fourier transform the resulting data stream;

3. Apply the low-pass filter $W=I-N^{-1}$;

4. Inverse Fourier transform back to real space;

5. Co-add the resulting data stream into the map $\hat{y}^{n+1}$.

Assuming that the normalised noise power spectrum can be approximated by $P(f)=1+\left(f_{0} / f\right)^{\alpha}$, the low-pass filter associated to each relaxation step is given by

$W(f)=\frac{f_{0}^{\alpha}}{f_{0}^{\alpha}+f^{\alpha}}$.

Since both $A$ and $P$ are norm-preserving operators, the norm of the increment $\Delta \hat{y}^{n}=\hat{y}^{n}-\hat{y}^{n-1}$ between step $n$ and $n+1$ decreases as $\left\|\Delta \hat{y}^{n+1}\right\| \leq W\left(f_{\min }\right)\left\|\Delta \hat{y}^{n}\right\|$, where $f_{\min }$ is a minimal frequency in the problem. Since $W\left(f_{\min }\right)<1$, we see that the approximate Jacobi relaxation scheme will converge in every case, which is good news. On the other hand, since $W\left(f_{\min }\right) \simeq 1$, the actual convergence rate of the scheme is likely to be very, very slow, which is bad news (cf. Fig. 6 for a graphical illustration). The fact that this algorithm is robust, but dramatically slow is a well-known property of the Jacobi method. The multi-grid method is also well known to solve this convergence speed problem. Note that if the convergence is reached, the solution we get is the optimal solution, i.e. similar to the one that would be obtained by a full matrix inversion up to round-up errors.

\subsubsection{Multigrid relaxation}

The multi-grid method for solving linear problems is described in great detail in Press et al. (1992). At each relaxation step at level $k=k_{\max }$, our target resolution, we can define the error $e_{k}^{n}=\hat{y}_{k}^{n}-\hat{y}_{k}$ and the residual $r_{k}^{n}=M_{k} \hat{y}_{k}^{n}-b_{k}$. Both are related through

$M_{k} e_{k}^{n}=r_{k}^{n}$.

If we are able to solve exactly for Eq. (23), the overall problem is solved. The idea of the multi-grid algorithm is to solve approximately for Eq. (23) using a coarser "grid" at resolution $k-1$, where the relaxation scheme should converge faster. We thus need to define a fine-to-coarse operator, in order to define the new problem on the coarse grid and solve for it. We also need a coarse-to-fine operator in order to inject the solution onto the fine grid. The approximation to the error $e_{k}^{n}$ is finally added to the solution. The coarse grid solver is usually applied after a few iterations on the fine level have been performed (in practice we perform 3 to 5 iterations). Naturally, since the solution to the problem on the coarse level relies also on the same relaxation scheme, it can be itself accelerated using an even coarser grid. This naturally leads to a recursive approach of the problem.

We defined our fine-to-coarse operator to be an averaging of the values of the 4 fine pixels contained in each coarse pixel. The coarse-to-fine operator is just a straight injection of the value of the coarse pixel into the 4 fine pixels. The most important issue is the temporal rebinning of the data stream, since the speed of the iterative scheme at a given level is actually set by the two Fourier transforms. We performed that resampling by simply taking one point out of two each time we go up one level. At the lower resolutions, this reduction is such that the iteration cost is negligible when compared to that at higher $k$; it allows fast enough iterations that full convergence may be reached. In practice, we choose a minimal level $k=3$ defined by $N_{\text {side }}=8$ and iterate a few hundred times to reach exact convergence.

Finally, the navigation through levels allows several options to be taken. Either we go up and down through all the levels successively (the so-called "V-cycle") or we follow more intricate paths (e.g. the "W-cycle" where at a given level we go down and up all the lower levels before going up and vice-versa). Since it turns out that levels are relatively disconnected in the sense that the scales well 
solved at a given resolution are only slightly affected by the solution at a higher level, the "V-cycle" is appropriate and is the solution we adopt in the two following configurations.

\section{Practical application to ARCHEOPS and TopHat experiments}

We now aim at demonstrating the capabilities of this algorithm with simulated data whose characteristics are inspired by those of the ARCHEOPS and TopHat experiments.

\subsection{Simulation}

The ARCHEOPS and TopHat experiments are very similar with respect to their scanning strategy. Indeed both use a telescope that simply spins at a constant rate (respectively 3 and 4 RPM) about its vertical axis. Thus due to Earth rotation the sky coverage of both is performed through scan circles whose axis slowly drifts on the sky. Nevertheless, because of the different launch points (respectively on the Arctic circle (Kiruna, Sweden) and in Antarctica (McMurdo)) and their different telescope axis elevation (respectively $\sim 41^{\circ}$ or $12^{\circ}$ ) they do not have the same sky coverage.

Otherwise the two experiments do not use the same bolometers technology, the same bands or have the same number of bolometers. But even if we try to be fairly realistic, our goal though is not to compare their respective performances but rather to demonstrate two applications of our technique in different settings. We then simulate for each a data stream of $\sim 24 \mathrm{hrs}$ duration with respectively a sampling frequency of 171 and $64 \mathrm{~Hz}$. The TODs contain realistic CMB and Galactic signal for a band at $143 \mathrm{GHz}$. Note that this is a one day data stream for TopHat (out of 10 expected) and that this frequency choice is more appropriate for ARCHEOPS than for TopHat (whose equivalent band is around $156 \mathrm{GHz}$ ), but this is realistic enough for our purpose. We generated a Gaussian noise time stream with the following power spectrum: $P(f) \propto\left(1+\left(f_{\text {knee }} / f\right)^{\alpha}\right)^{-1}$ with $f_{\text {knee }}=0.24$ and $1 \mathrm{~Hz}$, and $\alpha=1.68$ and 1 . The noise amplitude per channel is chosen so that it corresponds for ARCHEOPS (24 hours) and TopHat ${ }^{3}$ (10 days of flight) to $30 / 8 \mu \mathrm{K}$ on average per $20^{\prime}$ pixel, with a beam $F W H M$ of $10^{\prime} / 20^{\prime}$. Note that we restrict ourselves arbitrarily to shorter timeline that the method could handle in principle for time saving reason only. In principle the method could possibly deal with the full timeline at once (see below for memory issue discussion in Sect. 4.2) for the two experiments but we restrict ourselves on purpose to mere demonstrative examples.

We introduced 5 distinct levels of resolution defined by their $N_{\text {side }}$ parameter in the HEALPix package (Górski et al. 1998). The highest resolution level is imposed by the

\footnotetext{
${ }^{3}$ Lloyd Knox private communication.
}

pixelisation noise level requirement (Sect. 2.2.3) to $N_{\text {side }}=$ 256 (pixel size $\sim 13.7^{\prime}$ ) whereas the lowest one is $N_{\text {side }}=$ 8 (pixel size $\sim 7.3^{\circ}$ ). Therefore these two configurations each offer an interesting test since they differ by the sky coverage and the noise power spectrum. We iterate 3 times at each level except at the lowest one where we iterate 100 times.

\subsection{Results}

The algorithm is equally efficient in both situations. Whereas for ARCHEOPS (whose timeline is longer due to the higher sampling frequency) it took 2.25 hours on a SGI ORIGIN 2000 single processor, it took around 1.5 hours for the TopHat daily data stream.

In Figs. 1 and 2 we depict from top to bottom and from left to right the initial co-added data, the reconstructed noise map, the hit map, i.e. the number of hits per pixel at the highest resolution, the initial signal map as well as the reconstructed signal map and the error map. We see that the destriping is excellent in both situations and the signal maps recovered only contain an apparently isotropic noise. We note the natural correlation between the error map and the hit map. Finally, we stress that no previous filtering at all has been applied.

As an illustration of how our multi-grid work method works, Fig. 3 shows how the noise map is reconstructed at various scales.

\subsection{Tests}

In this section, we present some tests we carried out to validate our method. First, as was stated below, as soon as the iterative algorithm converges, the solution is by construction the optimal solution, similar to the one that would be obtained by the full matrix inversion. As a criterium for convergence we required the 2-norm of residuals to be of the order of the machine accuracy.

We initially have a Gaussian random noise stream fully characterised by its power spectrum. Therefore an important test is to check wether the deduced noise stream (by "observing" the stripe map, see Sect. 4.3 for further details) is Gaussian and has the same power spectrum. In Fig. 4 we ensure that the evaluated noise time stream is indeed Gaussian. As depicted in Fig. 5, where we plot in the ARCHEOPS case both the analytical expression of the spectrum according to which we generate the timeline and its recovered form, the agreement is excellent. We recall that we assumed at the beginning a perfect knowledge of the noise in order to define the filters. This is naturally unrealistic but the issue of noise evaluation is discussed in Sect. 4.3 below. We plotted as well the probability distribution function (PDF) of the final error map, i.e. the recovered noisy signal map minus the input signal map (Fig. 4). This PDF is well fitted by a Gaussian whose parameters are given in Fig. 4. The PDF of the error map displays some non-Gaussian wings. Let us recall that this 
Coadded Map

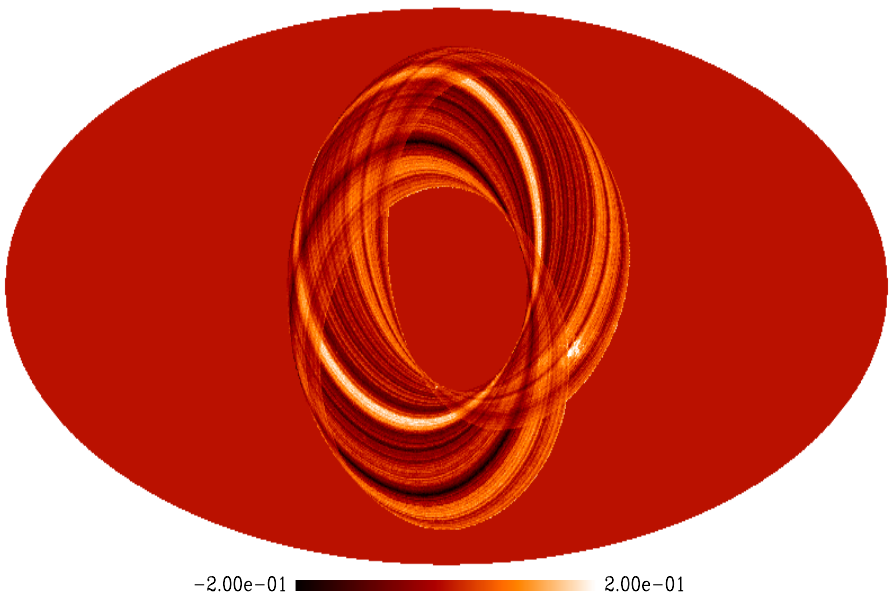

Recovered Noise Map

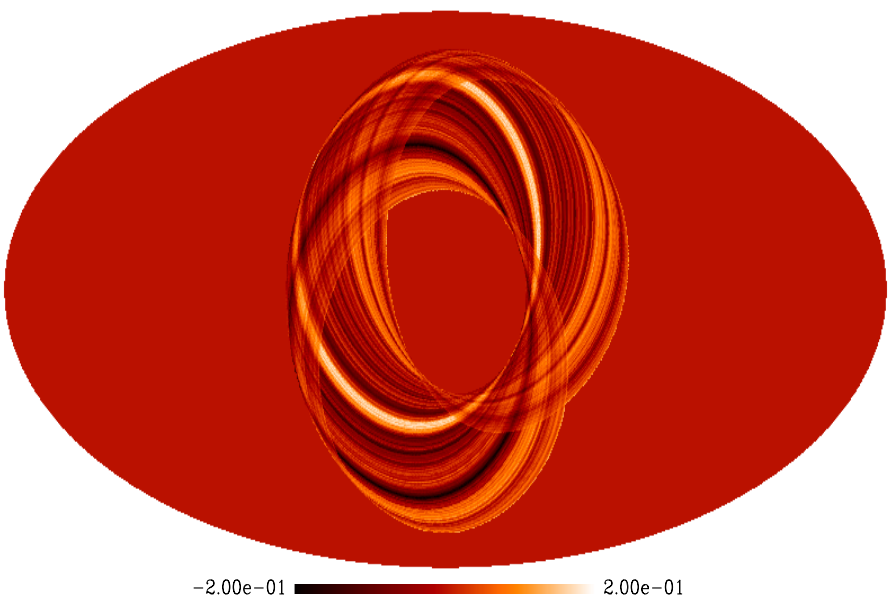

Hit Map

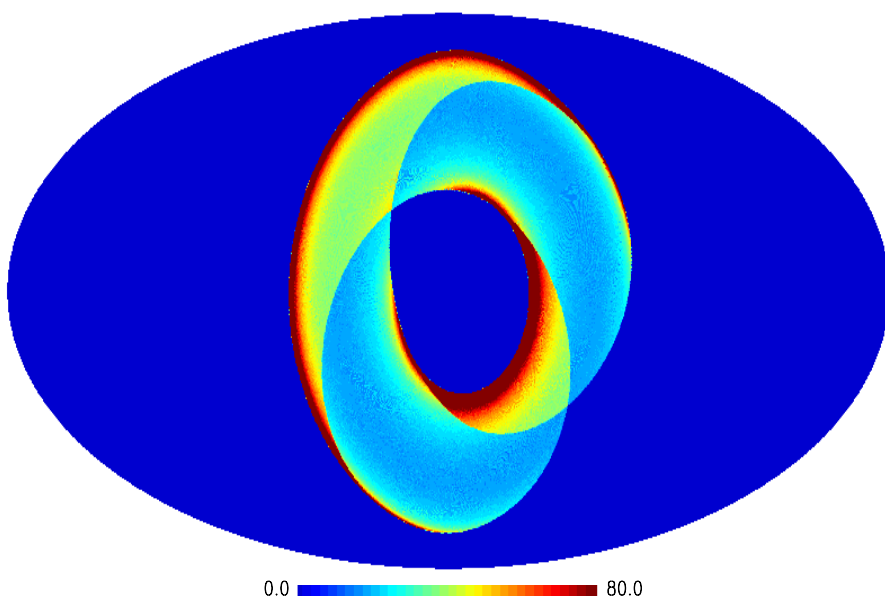

Signal

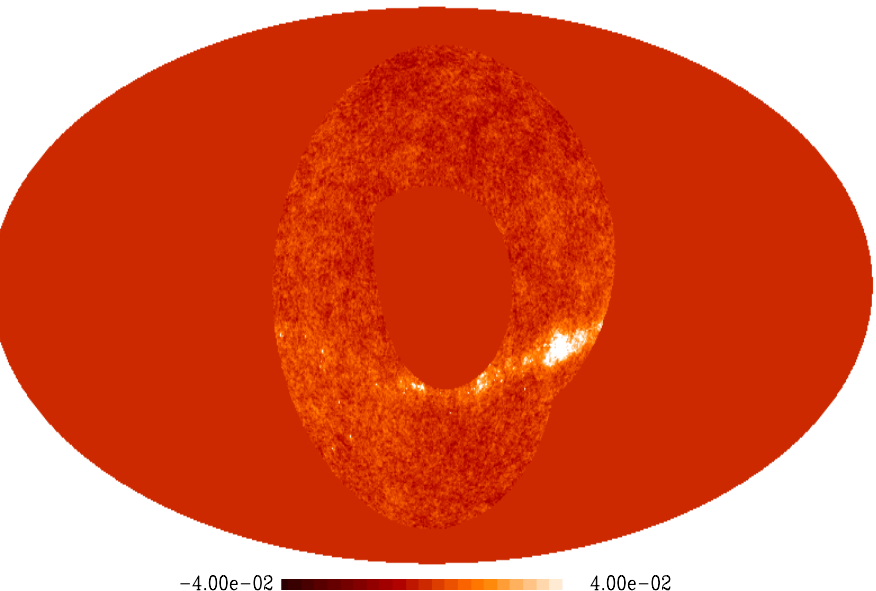

Recovered Signal Map

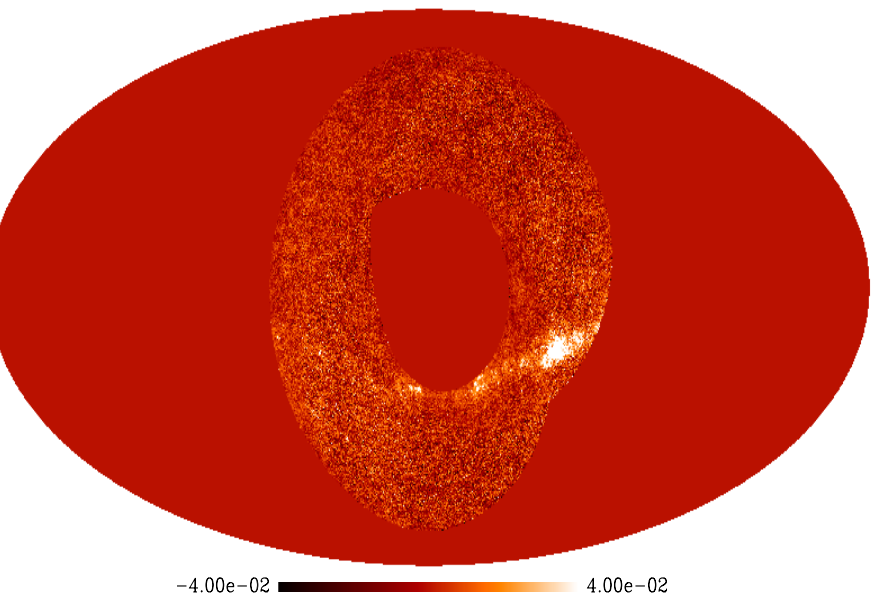

Error Map

Fig. 1. Simulated ARCHEOPS Kiruna flight: from top to bottom and from left to right, the co-added map and the input Galactic + CMB signal map, the reconstructed noise (stripes) and signal maps, the hit count and the error map (Arbitrary unit). The fact that the coverage is not fully symmetrical is due to the fact that we considered slightly less than $24 \mathrm{hr}$. Mollweide projection with pixels of $13.7^{\prime}$ (HEALPix $N_{\text {side }}=256$ ). Arbitrary units. 
Coadded Map

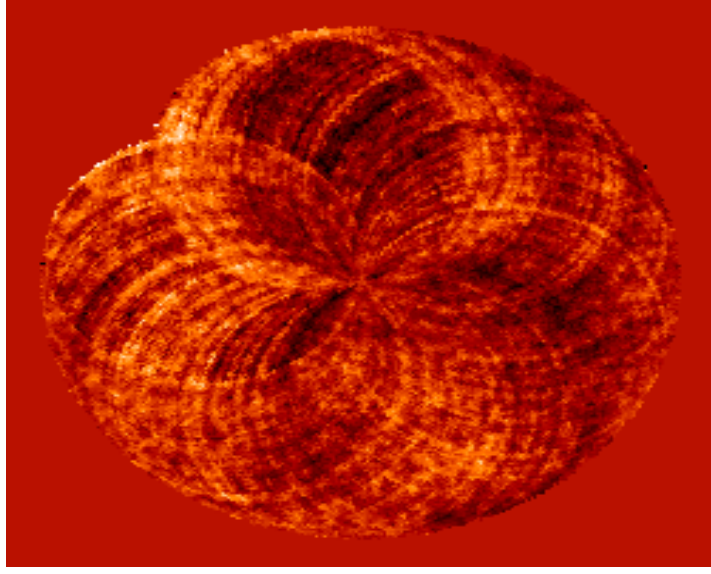

$-2.50 \mathrm{e}-02$

Recovered Noise Map

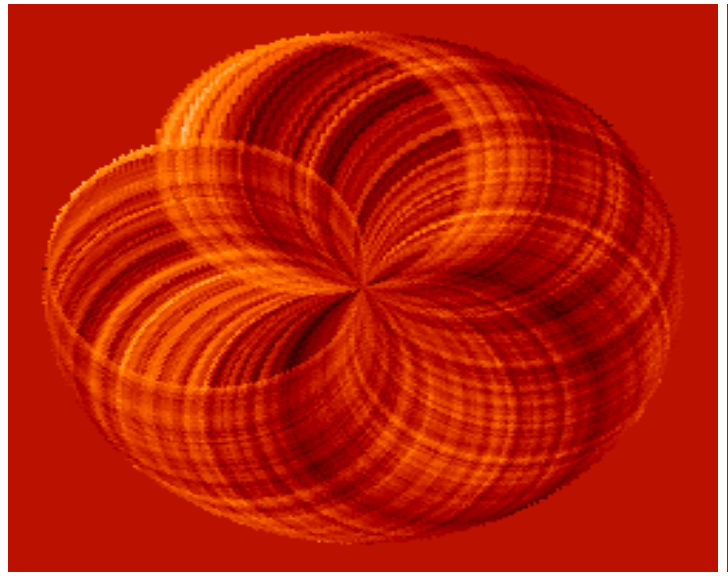

$(0.0,-90.0)$ Equatorial

Hit Map

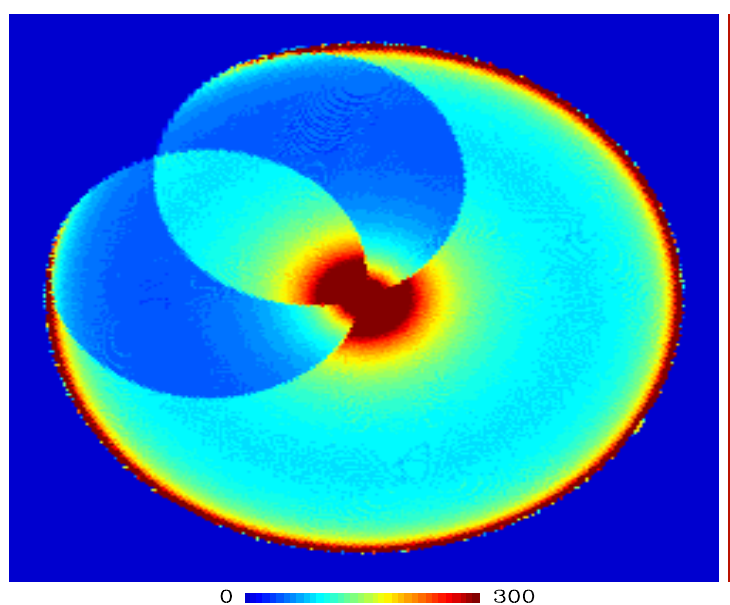

$(0.0,-90.0)$ Equatorial 300
Signal Map

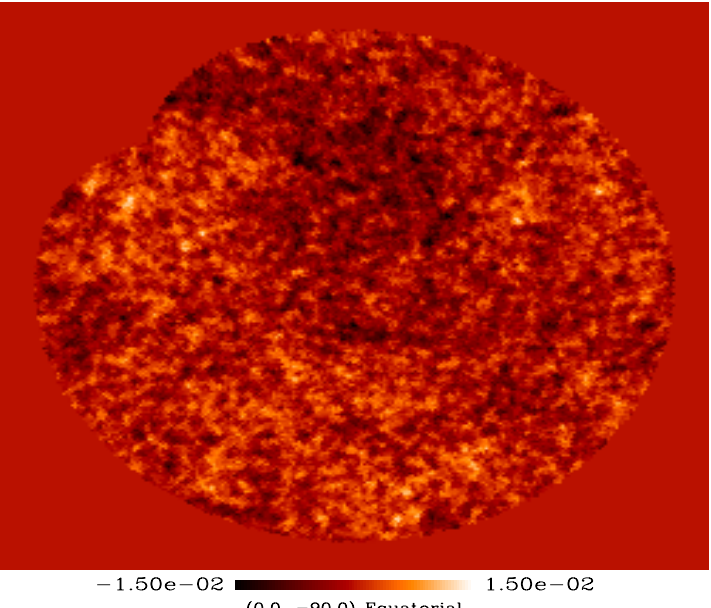

Recovered Signal Map

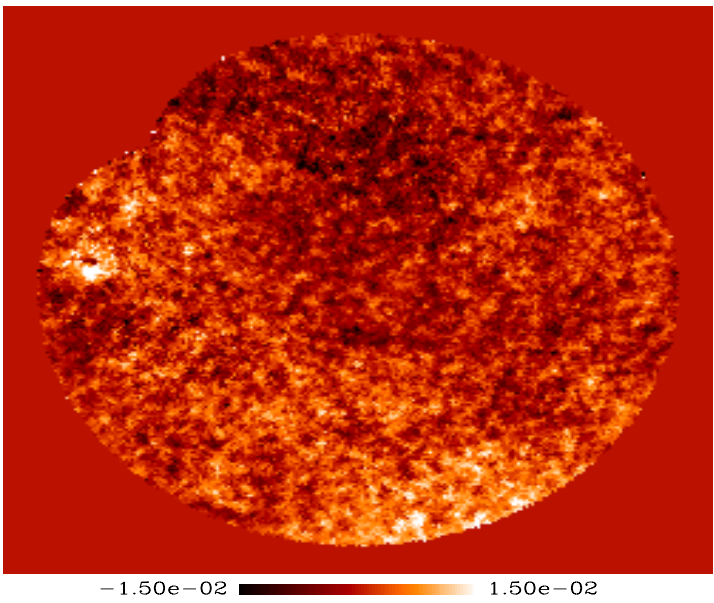

$(0.0,-90.0)$ Equatorial

Error Map

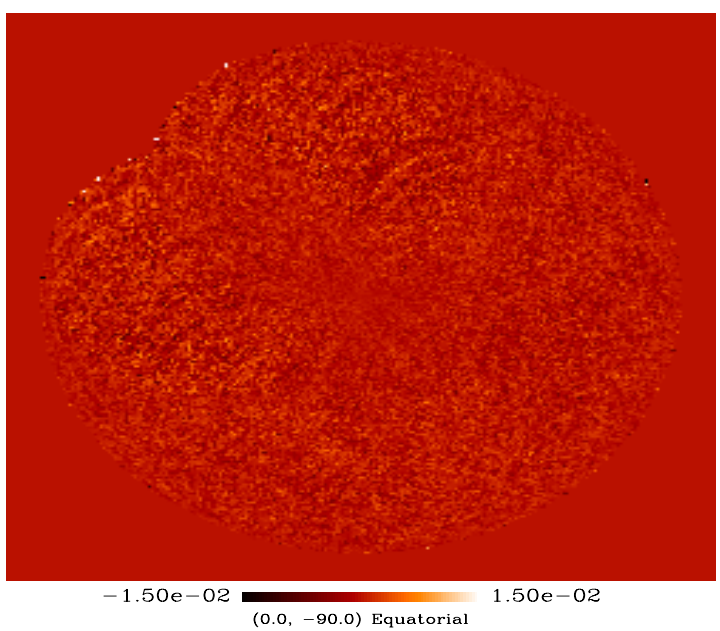

Fig. 2. Simulated TopHat one day flight: from top to bottom and from left to right, the co-added map and the input CMB + Galaxy signal map, the reconstructed noise (or stripes) and signal map, the hit count and error map. The fact that the coverage is not fully symmetrical is due to the fact that we consider only $18.2 \mathrm{hr}$ of flight. Gnomonic projection with pixel of $13.7^{\prime}$ (HEALPix $N_{\text {side }}=256$ ). Note that the slight visible striping is correlated to the incomplete rotation pattern that we arbitrary chose. Arbitrary units. 
Level 3

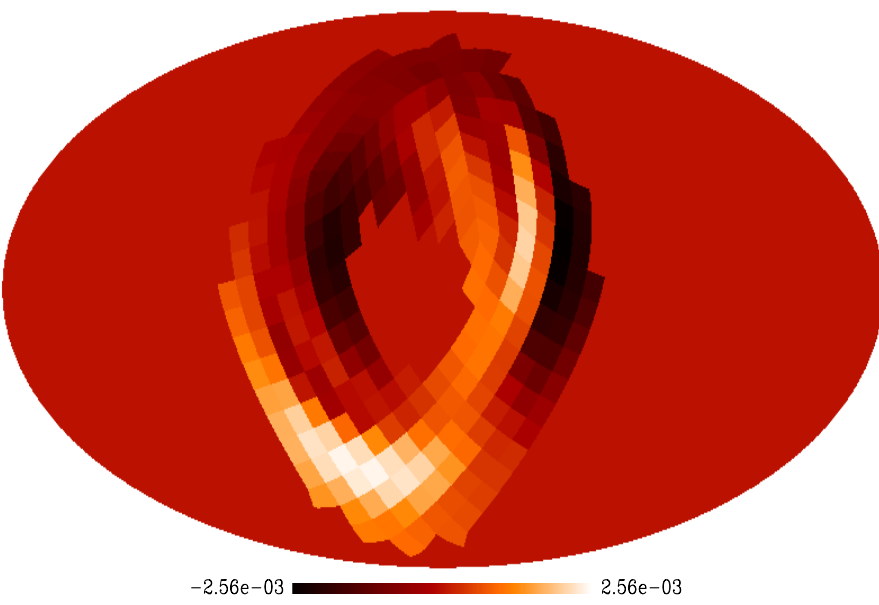

Level 5

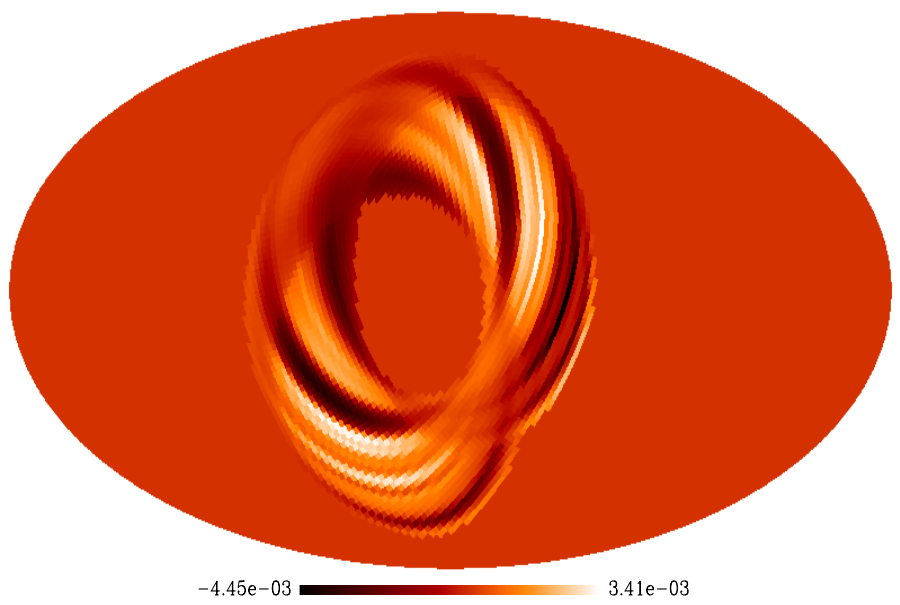

Level 4

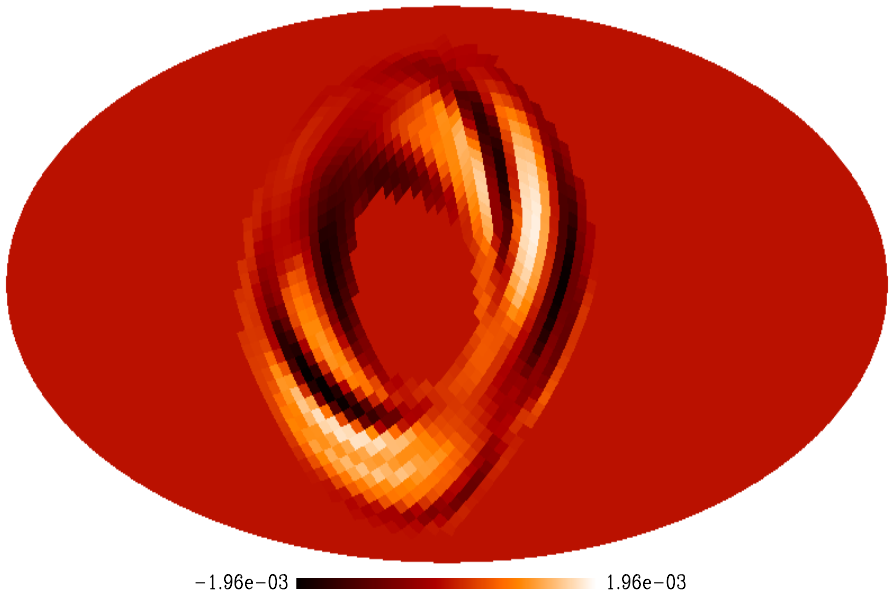

Level 6

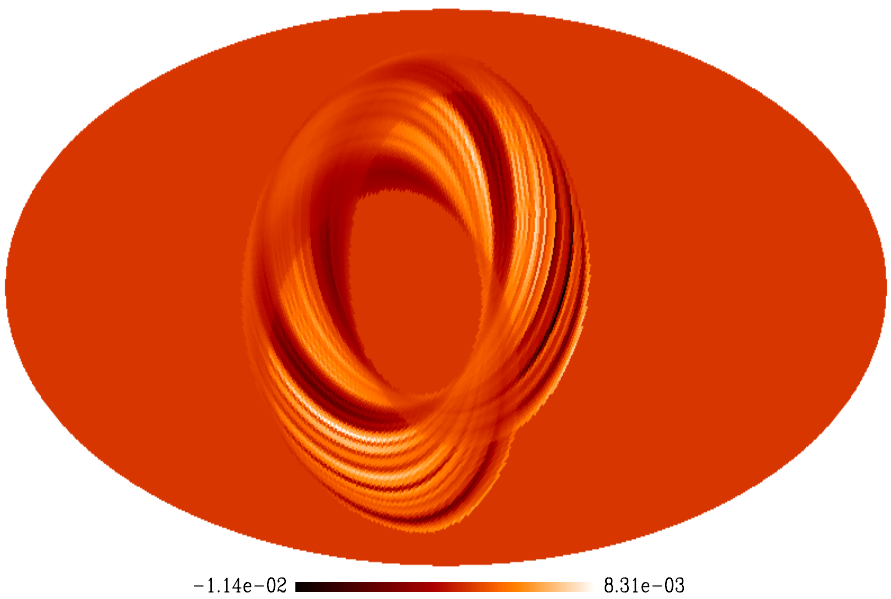

Fig. 3. Multi-grid noise map recovery: in this plot we show in the ARCHEOPS Kiruna case how the noise map is reconstructed at various levels, corresponding respectively to $N_{\text {side }}=8,16,32,64$.

is no surprise here because of the non-uniform sky coverage as well as the slight residual striping, both due to the non-ideal scanning strategy, i.e. that produces a nonuniform white noise in pixel space.

Another particularly important test consists in checking the absence of correlation between the recovered noise map and the initial signal map. We could not find any which is no surprise since we are iterating on a noise map (see Sect. 2.2.2) which does not contain any signal up to the pixelisation noise, that is ensured to be negligible with regards to the instrumental noise by choice of the resolution $k_{\max }$ (see Sect. 2.2.1).

\section{Discussion}

\subsection{Why is such an algorithm so efficient?}

The efficiency of such a method can be intuitively understood. Indeed, although the Jacobi method is known to converge very safely, it suffers intrinsically from a very slow convergence for large-scale correlations (which originate mainly in the off diagonal terms of the matrix to be inverted) (Press et al. 1992). This is illustrated in Fig. 6: we show the maps of residuals after solving this system using a standard Jacobi method on simulated data with 50, 100, 150, and 200 iterations. We used the same simulation and therefore the same sky coverage. Obviously the largest structures are the slowest to converge (implying observed large scale residual patterns). As a consequence it seems very natural to help the convergence by solving the problem at larger scales. Whereas large-scale structures will not be affected by a scale change, smaller structures will converge faster.

\subsection{Scalings}

We have found that this multi-grid algorithm translates naturally in a speed up factor greater than 10 as compared to a standard Jacobi method. This is illustrated 

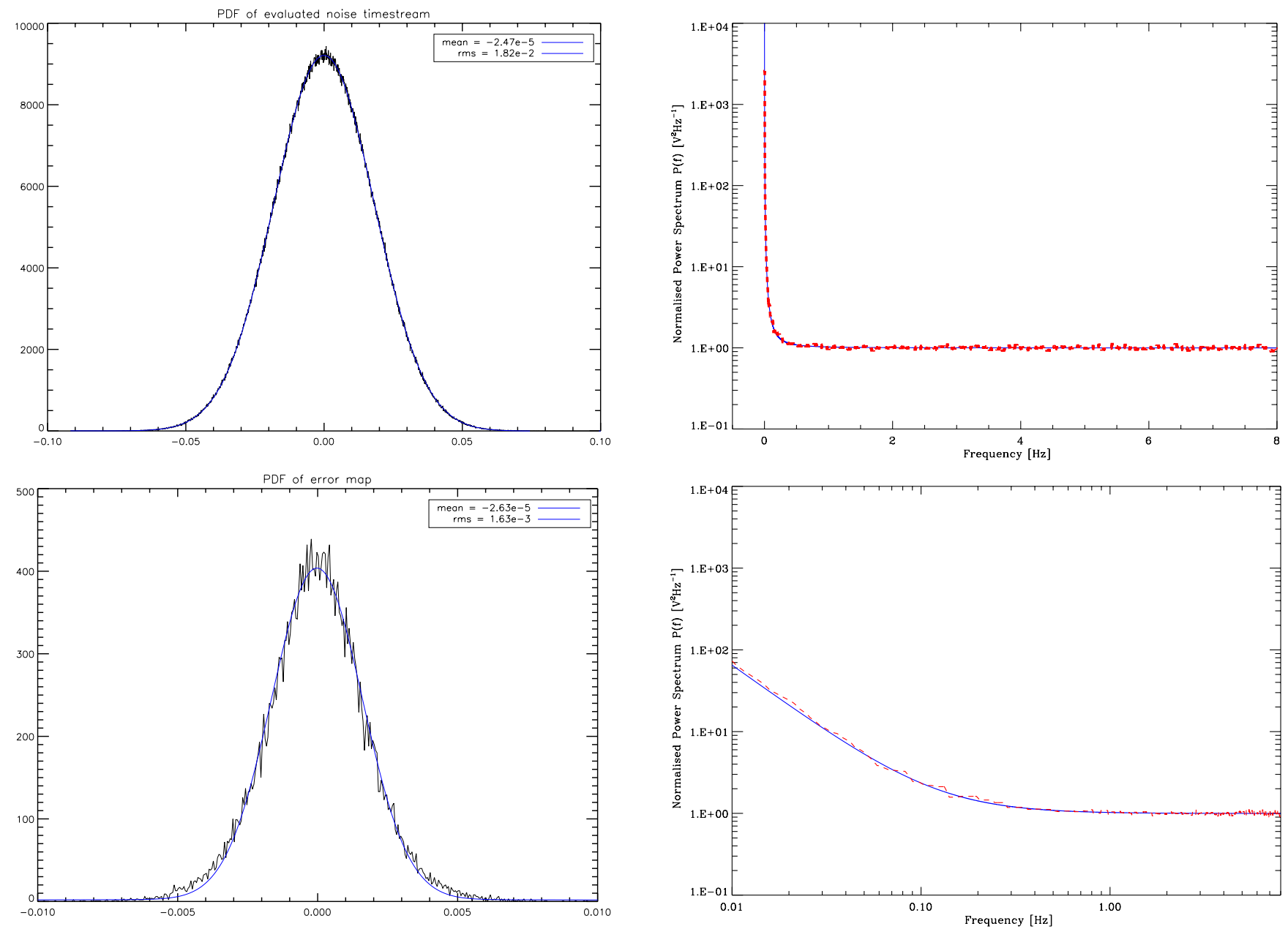

Fig. 4. In the TopHat case we plot from top to bottom the recovered probability distribution function of the noise stream evaluated along the timeline as well as the error map PDF. In these two cases a fit to a Gaussian has been performed whose parameters are written inside the figures. No significant departure from a Gaussian distribution are detected. The arbitrary units are the same as the ones used for the previously shown maps.

in Fig. 7 where we plotted the evolution of the 2-norm of residuals for the two methods in terms of the number of iterations in "cycle units". One cycle corresponds to 8 iterations at level $k_{\max }$ for a standard Jacobi method whereas it incorporates additionally going up and down all the lowest levels in the multi-grid case. Thus the cycle timing is not exactly the same but the difference is negligible since the limiting stages are definitely the iterations performed at maximum resolution. Note the fact that the efficiency of the multi-grid method allows us to solve exactly the system up to the 4-byte machine accuracy (small rebounds at the bottom of the curve) in approximately $135 \mathrm{mn}$ for $\left(\mathcal{N}_{\text {tod }} \sim 8 \times 10^{6}, \mathcal{N}_{\text {pix }} \sim 8 \times 10^{5}\right)$ on a SGI ORIGIN 2000 single processor. Note also that this ultimate convergence is definitely an overkill and is somewhat artificial for current CMB experiments. Indeed, residuals with a norm smaller than noise per pixel are

Fig. 5. Recovery of the noise power spectrum in the Archeops case (top: linear $x$-axis, bottom: $\log x$-axis): the red thin dashed line shows the initial analytic noise power spectrum used to generate the input noise stream and the blue thick line denotes the recovered one after 6 iterations. The recovered one has been binned as described in Sect. 4.3 and both have been normalised so that the white high frequency noise spectrum is equal to one. The agreement is obviously very good. No apparent bias is visible. Note that a perfect noise power spectrum prior knowledge has been used in this application.

meaningless. Since the limiting stages are the FFT's at the higher levels, this algorithm scales as $\mathcal{O}\left(\mathcal{N}_{\text {tod }} \ln \mathcal{N}_{\text {tod }}\right)$. In its current implementation, a rough scaling could be written this way:

$t=2.25$ hours $\frac{\mathcal{N}_{\text {tod }} \ln \left(\mathcal{N}_{\text {tod }}\right)}{12 \times 10^{6} \ln \left(12 \times 10^{6}\right)} \frac{N_{\text {cycle }}}{6}$

(note that we expect some further speed-up by reducing the number of operations at level max and eventually using a faster FFT). In terms of memory storage (RAM) it scales naturally as $\mathcal{O}\left(\mathcal{N}_{\text {tod }}\right)$ since one crucial feature of this iterative method is to handle only vectors and never an explicit matrix. Typically one single timeline size object is needed at any one time in memory. Since projection and observation operations are formally independent of the number of pixels, the scaling in $\mathcal{N}_{\text {pix }}$ is definitely 

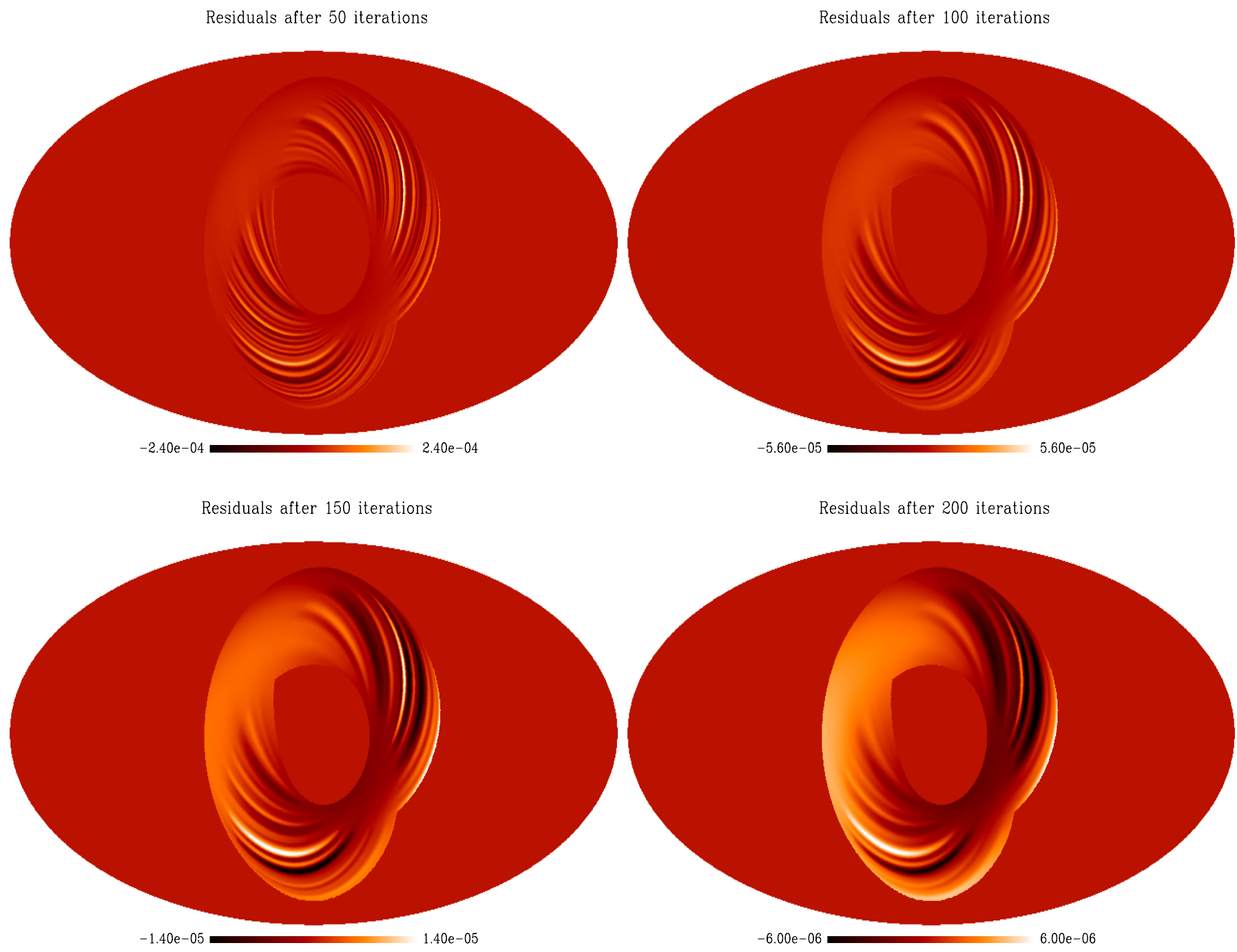

Fig. 6. Residual map after 50, 100, 150 and 200 iterations of a standard Jacobi method. This has been performed on simulated data for one bolometer with a nominal noise level. The sky coverage is that of ARCHEOPS coming Kiruna flight. The residual large scale patterns illustrate the difficulties the standard Jacobi method faces to converge. The stripes free area are the ones of scan crossing (see the hit map in Fig. 1).

subdominant. In terms of speed and efficiency, this method should well compare to a more obvious iterative inversion scheme such as preconditioned conjuguate gradient technique. The latter also has a scaling dominated by FFT as $\mathcal{O}\left(\mathcal{N}_{\text {tod }} \ln \mathcal{N}_{\text {tod }}\right)$ (Natoli et al.). A precise study comparing the relevant pre-factors but also discussing convergence criteria will be the object of a forthcoming paper.

\subsection{The noise estimation issue and noise covariance matrix estimation}

The estimation of the statistical properties of the noise in the timestream is an issue of paramount importance for map-making. Indeed, whereas till now we have assumed a perfect prior knowledge of the noise power spectrum in order to define the filters, it might not be that easy in practice since we cannot separate the signal from the noise.
We will therefore aim at making a joint estimation of the signal and the noise. This has been pioneered recently by Ferreira \& Jaffe (2000) and implemented independently by Prunet et al. (2000). The latter implementation is rather straightforward in our particular case since it just implies reevaluating the filters after a number of iterations, given the (current) estimation of the signal map and thus of the signal data stream. Nevertheless its non-obvious convergence properties have to be studied carefully through simulations. Making use of (17) our evaluation of the noise timeline $\hat{n}^{n}$ at the $n$th iteration and at level max is

$\hat{n}^{n}=d-A\left(\hat{y}_{k_{\max }}^{n}+P d\right)$.

Then we compute its spectrum and (re-) define the required filters. We then go through several multi-grid cycles ( 5 in the above demonstrated case) before reevaluating the noise stream. Very few evaluations of the noise are needed before getting a converged power 
spectrum (around 2). In such an implementation, no noise priors at all are assumed. This is illustrated on one particular worked out example in the case of a 4 hours ARCHEOPS like flight (more detailed considerations will be discussed elsewhere). To reduce the number of degrees of freedom we bin the evaluated noise power spectrum using a constant logarithmic binning $(\Delta \ln f=0.15$ in our case) for $f \leq 2 f_{\text {knee }}$ and a constant linear binning ( $\Delta f=0.08 \mathrm{~Hz}$ in our case) for higher frequency. Figure 8 shows the genuine and evaluated noise power spectrum. The initial noise power spectrum was a realistic one $P(f) \propto\left(1+\left(f_{\text {knee }} / f\right)^{\alpha}\right)$ to which we added some small perturbations (the two visible bumps) to test the method. Note the small bias around the telescope spin frequency at $f_{\text {spin }}=0.05 \mathrm{~Hz}$ : this is illustrative of the difficulties we fundamentally face to separate signal and noise at this particular frequency through Eq. (17). Naturally, this bias was not present in the case demonstrated in Fig. 5 where we assumed a prior knowledge of the spectrum. This possible bias forced us to work with a coarser binning $(\Delta \ln f=1$.) in the $1 / f$ part of the spectrum until the convergence is reached, i.e. we evaluate the noise power spectrum with the previously mentioned binning only at the last step. Proceeding this way, the convergence towards the correct spectrum is both fast (3 noise evaluations) and stable.

Second, the output of any map-making is meaningless without an evaluation of the map noise covariance matrix $\left(A^{T} N^{-1} A\right)^{-1}$. Given such a fast algorithm (and the fact it gives an evaluation of the power spectrum), it is natural to obtain it through a Monte-Carlo algorithm fueled by various realizations of the noise timeline generated using the evaluated power spectrum (in the spirit of Wandelt et al. 2000). A detailed study will be performed in a future work.

However we investigate here the chance of this approach being successfull and illustrate it very briefly by a very rough $C_{\ell}$ determination (which is in no way an appropriate $C_{\ell}$ estimate). To this end we perform a one day TopHat like simulation including only the CMB signal plus the noise. From this data stream we obtain an "optimal" signal map as well as an evaluation of the noise power spectrum using the previously described algorithm. With the help of the anafast routine of the HEALPix package we calculate this way a rough $C_{\ell}^{\text {signal }}$. Using the estimated noise power spectrum we generate 10 realisations of the noise and get consequently 10 "optimal" noise maps. For each of them we measure as before $C_{\ell}$ and average them to obtain $C_{\ell}^{\text {noise }}$. In order to "de-bias" the signal power spectrum recovered in this way, we substract $C_{\ell}^{\text {noise }}$ from $C_{\ell}^{\text {signal }}$. The power spectrum obtained in this manner includes as well some spatial correlations due to weak residual stripping and thus does not correspond exactly to white noise (at least in the low $\ell$ part). For the sake of comparison we compute the power spectrum of the input signal measured the same way, $C_{\ell}^{\text {input }}$, and plotted both $C_{\ell}^{\text {input }}$ and $C_{\ell}^{\text {signal }}-C_{\ell}^{\text {noise }}$ averaged in linear bands

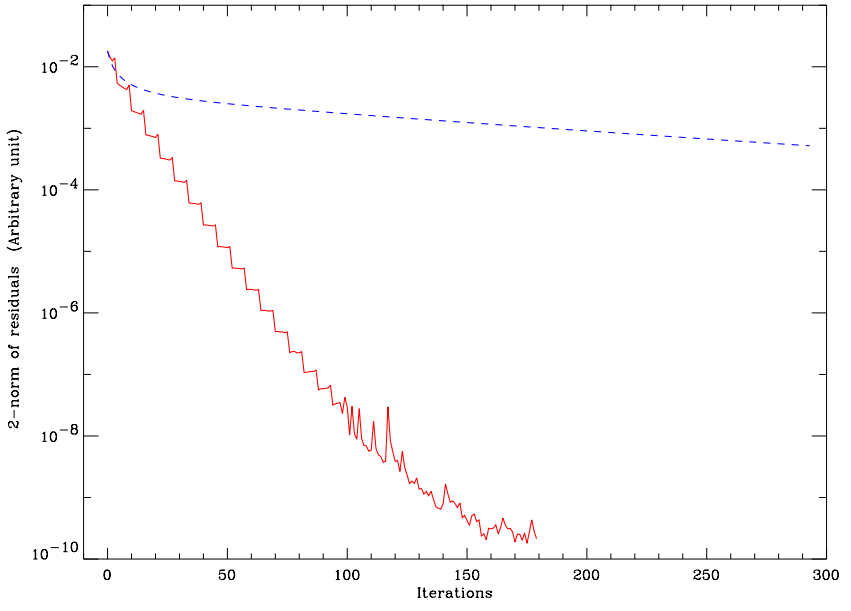

Fig. 7. The evolution of the 2-norm of residuals with the number of iterations at level max. Whereas the blue dashed line is standard iterative Jacobi, the solid red line is the iterative multi-grid Jacobi method. A full multi-grid cycle incorporates 3 iterations at level max before going down and up all the levels. The sharp jumps correspond to the moment when the scheme again reaches max level and thus benefits from the resolution at lower levels. Note that the very sharp features after $\sim 100$ iterations are due to the fact we reached the 4-byte machine accuracy which is almost out of reach for a standard iterative method.

of constant width $\Delta \ell=80$. The agreement is obviously very good, as illustrated in Fig. 9. The error bars take into account both the sampling induced variance as well as the beam spreading (Knox 1995).

This procedure is in no way an appropriate $C_{\ell}$ measurement since we are not dealing properly here with the sky coverage induced window function which triggers some spurious correlations within the $C_{\ell}$ 's. We thus do not take into account the full structure of the map noise covariance matrix. Nevertheless, the efficiency of such a rough method is encouraging for more detailed implementation and a full handling of the noise covariance matrix. Note finally that this is a naturally parallelised task which should therefore be feasible very quickly.

\subsection{Application to genuine data and hypothesis}

The application to genuine data could be problematic if our key hypotheses were not fulfilled. We now turn to their discussion. Concerning the noise, we assumed that it is Gaussian (in order to derive our map estimator) and stationary (in order to exploit the diagonality of its noise covariance matrix in Fourier space). Both hypotheses are reasonable for a nominal instrumental noise, at least on (sufficiently long) pieces of timeline. If not, we would have to cut the "bad" parts and replace them by a constrained realization of the noise in these parts. Concerning the signal, no particular assumptions are needed in the method we are presenting. At this level, we neglected as well the effect of the non perfect symmetry of the instrumental beam. This effect should be quantified for a real 

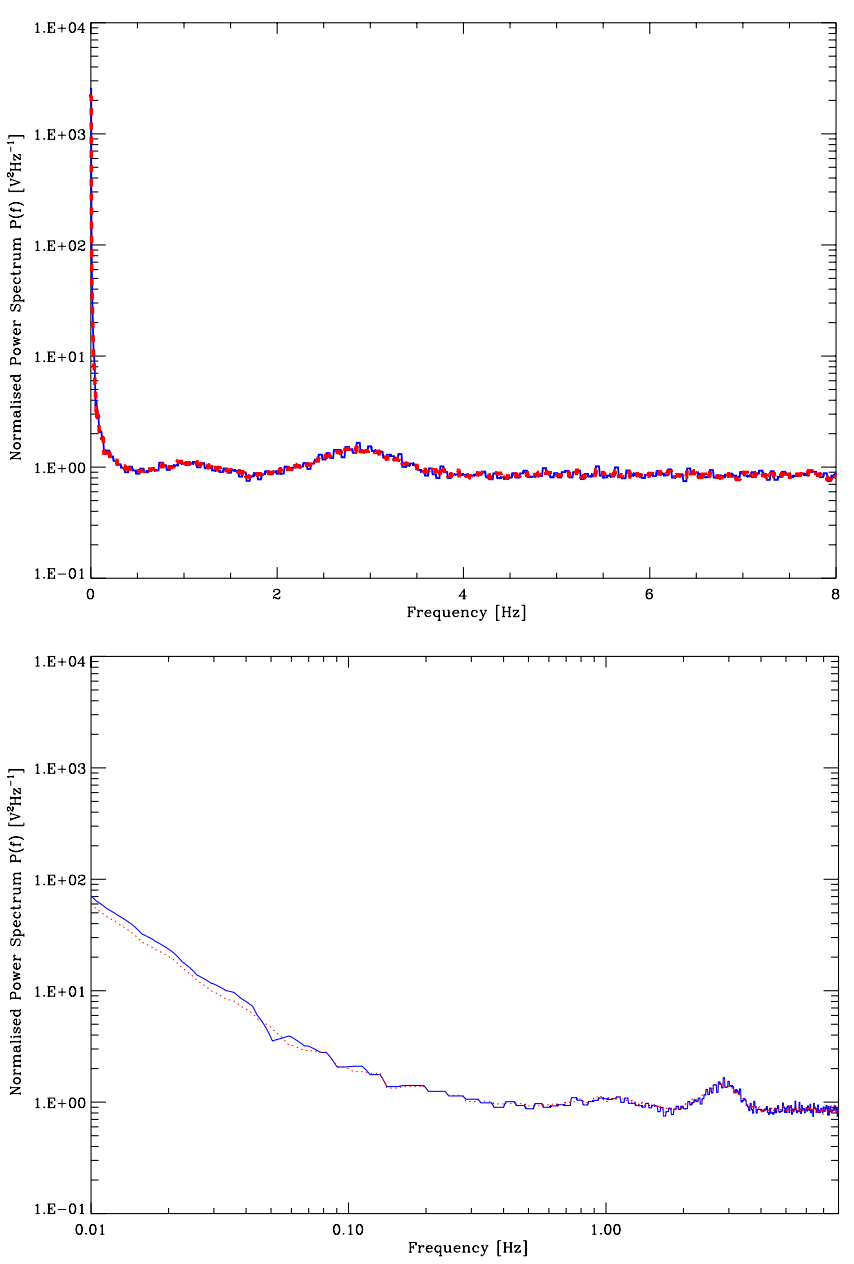

Fig. 8. Evaluation of the noise power spectrum (top: linear $x$-axis, bottom: $\log x$-axis): the red thin dashed line shows the initial noise power spectrum obtained from the input noise stream and the blue thick line denotes the recovered one after 5 iterations. Both have been smoothed and normalised so that the white high frequency noise spectrum is equal to one. The agreement is obviously very good. Note that no noise priors at all have been used in this evaluation.

experiment (Wu et al. 2000; Doré et al. 2000). Another technical hypothesis is the negligibility of the pixelisation noise with respect to the instrumental noise but since this is fully under control of the user it should not be a problem.

In this paper, we have presented an original fast mapmaking method based on a multi-grid Jacobi algorithm. It naturally entails the possibility of a joint noise/signal estimation. We propose a way to generate the noise covariance matrix and illustrate its ability on a simplistic $C_{\ell}$ estimation. The efficiency of this method has been demonstrated in the case of two coming balloon experiments, namely ARCHEOPS and TopHat but it naturally has a more general range of application. This tool should be of natural interest for Planck and this will be demonstrated elsewhere. Furthermore, due to the analogy of the formalisms, this should have some applications as well in the component separation problem.

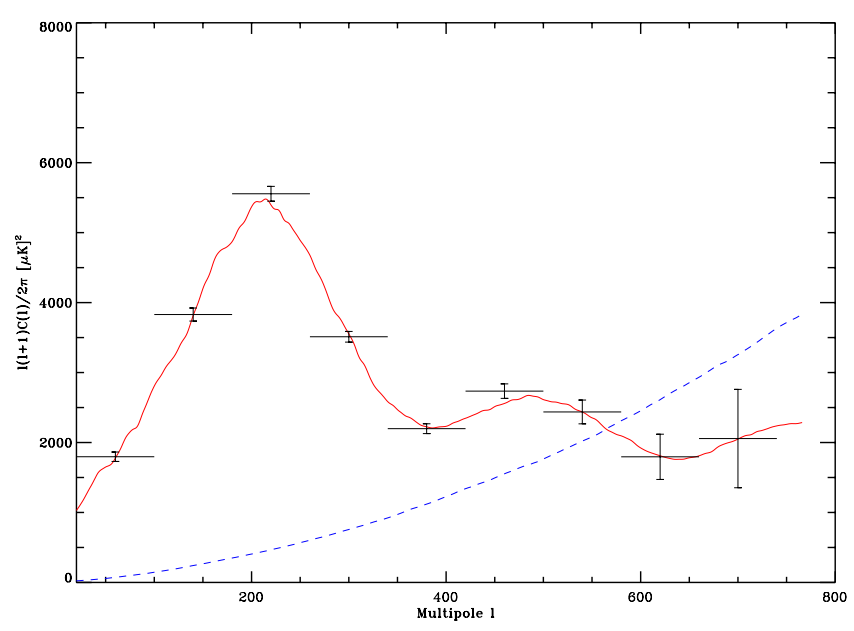

Fig. 9. In the case of a one day flight of the TopHat experiment we perform a very approximate evaluation of the recovered signal band powers (black) which has to be compared to the input signal power spectrum (red line). Both have been measured the same way using the anafast routine of the HEALPix package. These band powers $(\Delta \ell=80)$ have been performed using a fast Monte-Carlo evaluation of $C_{\ell}^{\text {noise }}$ (dashed blue line) which does not correspond exactly to white noise since there remains some spatial correlations. This in no way constitutes an appropriate $C_{\ell}$ measurement but is an encouraging step towards a full Monte-Carlo treatment.

We hope to apply it very soon to ARCHEOPS data. The FORTRAN 90 code whose results have been presented is available at http://ulysse. iap.fr/download/mapcumba/.

Acknowledgements. We would like to thank Eric Hivon for fruitful remarks, Lloyd Knox for stimulating discussions and information on the TopHat experiment, the developers of the HEALPix (Górski et al. 1998) package which has considerably eased this work and Julian Borrill, our referee for suggesting some valuable precisions.

\section{References}

Benoit, A., et al. 2000, submitted to A\&A

Bond, J. R., Jaffe, A. H., \& Knox, L. 1998, Phys. Rev. D, 2117 Bond, J. R., Crittenden, R. G., Andrew, H., Jaffe, A. H., \& Knox, L. 1999, Computing in Science and Engineering [astro-ph/9903166]

Borrill, J. 1999, MADCAP: The Microwave Anisotropy Dataset Computational Analysis Package Julian Borrill in Proceeedings of the 5th European SGI/Cray MPP Workshop [astro-ph/9911389], see also http://cfpa. berkeley.edu/ borrill/cmb/madcap.html

Borrill, J. 2000, in proceedings to the MPA/ESO/MPE Joint Astronomy Conference Mining the sky, Garching

Bunn, E. F., Hoffman, Y., \& Silk, J. 1996, ApJ, 464, 1

Bouchet, F. R. 1996, Gispert, AIP Conf. Proc. 348, The $\mathrm{mm} / \mathrm{sub}-\mathrm{mm}$ Foregrounds and Future CMB Space Missions, ed. E. Dwek

Bouchet, F. R., \& Gispert, R. 1999, New Astron., 4, 443B

de Bernardis, P., et al. 2000, Nature (London) 404, 995

Doré, O., Bouchet, F. R., Teyssier, R., \& Vibert, D. 2000, in proceedings to the MPA/ESO/MPE Joint Astronomy Conference Mining the sky, Garching 
Ferreira, P. G., \& Jaffe, A. H. 2000, MNRAS, 312, 89

Górski, E. K., Hivon, E., \& Wandelt, B. D. 1998, in proceedings of the MPA/ESO Garching Conf., ed. A. J. Banday, K. Sheth, \& L. Da Costa

http://www .eso.org/ kgorski/healpix/

Hanany, S., et al. 2000, ApJL, submitted [astro-ph/0005123]

Jansen, D. J., \& Gulkis, S., Mapping the Sky with the COBEDMR, in The Infrared and Submillimeter Sky after COBE, ed. M. Signore, \& C. Dupraz (Dordrecht: Kluwer)

Knox, L. 1995, Phys. Rev. D, 52, 4307

Natoli et al., A\&A, submitted [astro-ph/0101252]

Press, W. H., Teukolsky, S. A., Vetterling, W. T., \& Flannery, B. P. 1992, Numerical Recipes, 2nd ed. (Cambridge University Press, Cambridge)

Prunet, S., Netterfield, C. B., Hivon, E., \& Crill, B. P. 2000, Iterative map-making for scanning experiments, to be published in the Proceedings of the XXXVth Rencontres de Moriond, Energy Densities in the Universe (Éditions Frontières) [astro-ph/0006052]

Tegmark, M. 1997, ApJL, 480, L87

Tegmark, M., \& Efstathiou, G. 1996, MNRAS, 281, 1297

Wandelt, B. D., Hivon, E., \& Gorski, K. M., preprint [astro-ph/0008111]

Wandelt, B. D., \& Gorski, K. M., preprint [astro-ph/0008227]

$\mathrm{Wu}$, J. H. P., et al., ApJS, in press [astro-ph/0007212]

Wright, E. L., Hinshaw, G., \& Bennett, C. L. 1996, ApJL, 458, 53

Wright, E. L., proceeding of the IAS CMB Workshop [astro-ph/9612006]

Zaroubi, S., Hoffman, Y., Fisher, K. B., \& Lahav, O. 1995, ApJ, 449, 446 